CRYSTALLOGRAPHIC COMMUNICATIONS

ISSN 2056-9890

Received 29 June 2018

Accepted 25 July 2018

Edited by J. Ellena, Universidade de Sâo Paulo, Brazil

₹ Additional correspondence author, e-mail: Chem_sq@163.com.

Keywords: crystal structure; carbapenem; antibiotics; tebipenem; prodrug; hydrogen bonding.

CCDC reference: 1816052

Supporting information: this article has supporting information at journals.iucr.org/e

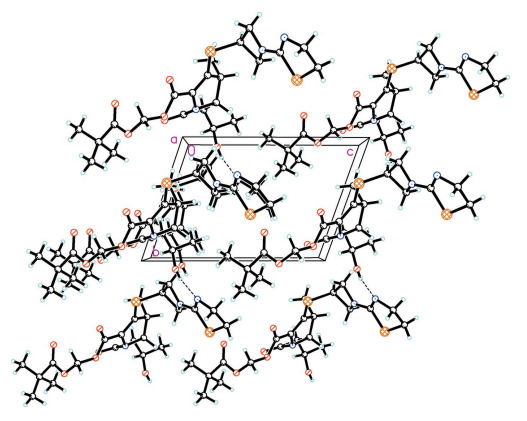

OPEN $\odot$ ACCESS

\section{Crystal structure of tebipenem pivoxil}

\author{
Chao Tang, ${ }^{a}$ Li Cai, ${ }^{b} *$ Shuai Liu, ${ }^{a}$ Zhiwei Zheng, ${ }^{a}$ Gen Li, ${ }^{a}$ Jianli Chen ${ }^{a}$ and Qiang \\ Sui ${ }^{\mathrm{a}} \neq$
}

${ }^{a}$ China State Institute of Pharmaceutical Industry, 285 Gebaini Rd, Shanghai 201203, People's Republic of China, and b University of South Carolina Lancaster, 476 Hubbard Drive, Lancaster, SC 29720, USA. *Correspondence e-mail: CAILI@mailbox.sc.edu

The molecular structure of the first orally active carbapenem antibacterial agent, tebipenem pivoxil (systematic name: (2,2-dimethylpropanoyloxy)methyl $(4 R, 5 S, 6 S)$-3-\{[1-(4,5-dihydro-1,3-thiazol-2-yl)azetidin-3-yl]sulfanyl $\}-6-[(1 R)$ 1-hydroxyethyl]-4-methyl-7-oxo-1-azabicyclo[3.2.0]hept-2-ene-2-carboxylate), $\mathrm{C}_{22} \mathrm{H}_{31} \mathrm{~N}_{3} \mathrm{O}_{6} \mathrm{~S}_{2}$, has been determined and the configurations of the four chiral centers validated. The title compound crystallizes in the triclinic space group $P 1$ with one molecule in the unit cell. Three out of the four rings adopt planar conformations while the thiazolinyl ring adopts an enveloped conformation. In the crystal, $\mathrm{O}-\mathrm{H} \cdots \mathrm{N}$ hydrogen bonds link the molecules into chains along $[1 \overline{1} 0]$.

\section{Chemical context}

Carbapenem antibiotics, like all $\beta$-lactam antibacterials that bind to and inhibit the peptidoglycan cross-linking transpeptidases, have attracted increasing attention recently because of their broader spectrum activities and stronger bactericidal actions compared to cephalosporins and penicillins. Since the first carbapenem structure thienamycin, a natural product derived from Streptomyces cattleya, was isolated in 1976 (Johnston et al., 1978), a handful of subsequent parenteral carbapenem agents, such as imipenem, panipenem, meropenem, biapenem, have been developed based on this parent compound and used clinically for the treatment of severe bacterial infections.<smiles>C[C@H]1C(SC2CN(C3=NCCS3)C2)=C(C(=O)OCOC(=O)C(C)(C)C)N2C(=O)[C@H](O)[C@H]12</smiles>

Tebipenem pivoxil (see scheme), as a novel oral carbapenem agent, was approved by the Pharmaceuticals and Medical Devices Agency of Japan (PMDA) on Apr 22, 2009. It was developed and marketed as Orapenem $\AA$ by Meiji Seika in Japan (as of 05/16/2016, the only approved country/area for its usage was Japan for treating children, as these oral antibiotics are often better tolerated than infusions) (Kijima et al., 2009). It is a prodrug that is quickly hydrolysed to the active antimicrobial agent LJC11,036 (5, reaction scheme) because the absorption rate of the pivaloyloxymethyl ester is higher than that of other prodrug-type $\beta$-lactam antibiotics (Kato et al., 2010). The active metabolite 5 shows potent and well- 
balanced antibacterial activity and also shows higher stability to human renal dehydropeptidase-I than meropenem (Isoda $e t$ al., 2006a; Kobayashi et al., 2005). Research has also revealed that the tebipenbem acyl- $\beta$-lactamase covalent complex remains very stable for longer than 90 min, partly explaining its resistance towards hydrolysis (Papp-Wallace et al., 2011).

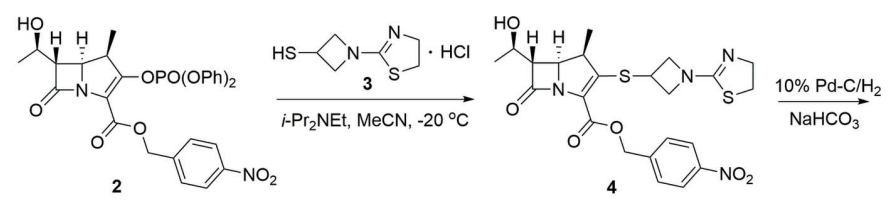

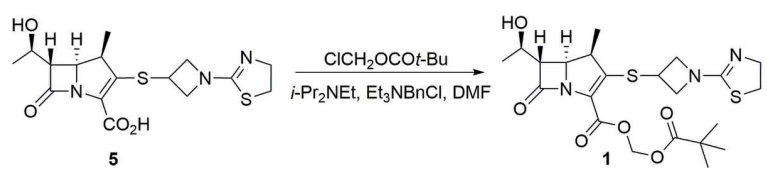

Tebipenem pivoxil has a complex structure with four chiral centers and a 1-(1,3-thiazolin-2-yl)azetidin-3-ylthio side chain at the $\mathrm{C}-2$ position. We hope the structural elucidation will facilitate future mechanistic studies of this molecule and of its interactions with enzymes that are responsible for bacterial resistance.

\section{Structural commentary}

Tebipenem pivoxil (Fig. 1) crystallizes in the triclinic space group $P 1$ with one molecule in the unit cell. The present crystal structure dertermination allowed the configurations of the four chiral centers to be validated as: $\mathrm{C} 2 S, \mathrm{C} 3 S, \mathrm{C} 4 R, \mathrm{C} 7 R$. Rings I (N1/C1-C3), II (N1/C3-C6) and III (N2/C11-C13) adopt planar conformations (with r.m.s. deviations of 0.0251 , 0.0838 , and $0.0967 \AA$, respectively) while ring IV (N3/S2/C14C16) adopts an envelope conformation with atom $\mathrm{C} 16$ as the flap. The dihedral angles between rings I and II, II and III, and III and IV are 46.7 (2), 85.7 (2), and $11.9(4)^{\circ}$, respectively. Atoms C9 (methyl) and C7 are located above and below the planes of rings I and II because of steric hindrance.

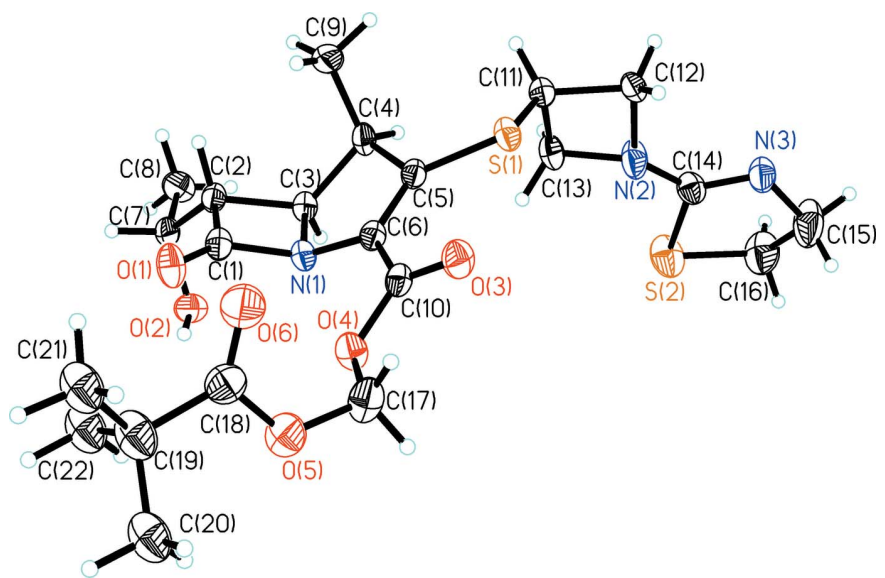

Figure 1

The molecular structure of the title compound, showing the atom labelling and $30 \%$ probability displacement ellipsoids.
Table 1

Hydrogen-bond geometry $\left(\AA{ }^{\circ}\right)$.

\begin{tabular}{lllll}
\hline$D-\mathrm{H} \cdots A$ & $D-\mathrm{H}$ & $\mathrm{H} \cdots A$ & $D \cdots A$ & $D-\mathrm{H} \cdots A$ \\
\hline $\mathrm{O} 2-\mathrm{H} 2 A \cdots \mathrm{N} 3^{\mathrm{i}}$ & 0.82 & 2.01 & $2.816(6)$ & 169 \\
$\mathrm{C} 11-\mathrm{H} 11 A \cdots \mathrm{O} 2^{\mathrm{ii}}$ & 0.98 & 2.43 & $3.366(6)$ & 160 \\
\hline
\end{tabular}

Symmetry codes: (i) $x-1, y+1, z$; (ii) $x, y-1, z$.

\section{Supramolecular features}

In the crystal, $\mathrm{O}-\mathrm{H} \cdots \mathrm{N}$ hydrogen bonds (Table 1) link the molecules into chains along [110]. $\mathrm{C}-\mathrm{H} \cdots \mathrm{O}$ hydrogen bonds are also observed. The packing viewed along the $a$ axis is shown in Fig. 2.

\section{Database survey}

The tebipenem pivoxil we obtained was well characterized spectroscopically and carefully compared with reference values (Isoda et al., 2006a). To the best of our knowledge, including a search of the Cambridge Structural Database (CSD Version 5.39; Groom et al., 2016), no single crystal structure determination has previously been reported for this drug.

\section{Synthesis and crystallization}

As shown in the reaction scheme (also see Supporting Information), 3-mercapto-1-(1,3-thiazolin-2-yl)-azetidine hydrochloride (3) was first synthesized according to a method previously reported (Isoda et al., 2006b) with minor optimizations. The side chain $\mathbf{3}$ was then coupled with the commercially available carbapenem core (2), followed by hydrogenation/deprotection and $\mathrm{SN}_{2}$ esterification to afford the desired tebipenem pivoxil 1 (Isoda et al., 2006a,b). Instead

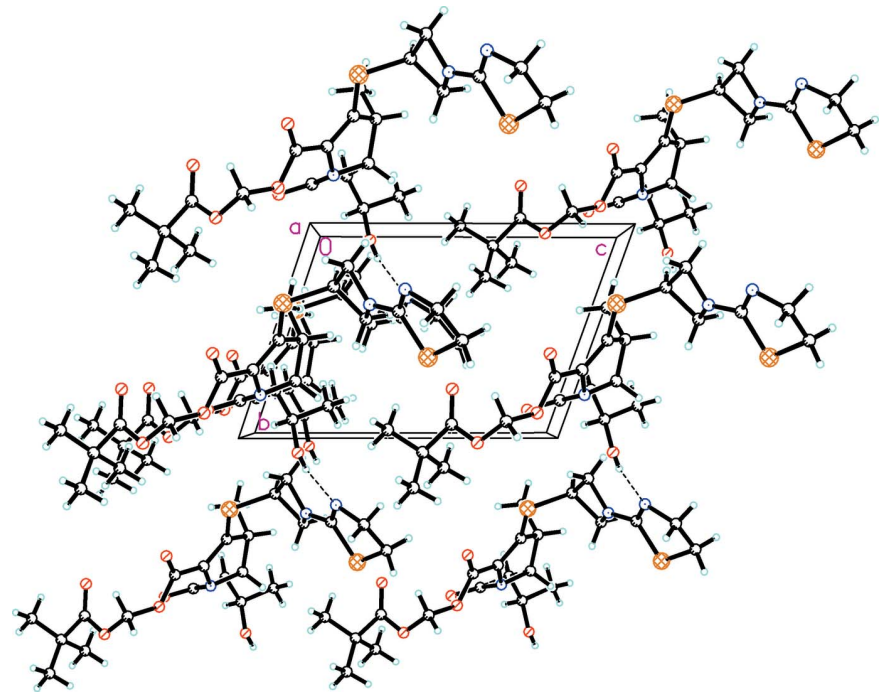

Figure 2

The crystal packing viewed along the crystallographic $a$ axis showing the $\mathrm{O}-\mathrm{H} \cdots \mathrm{N}$ hydrogen bonds (Table 1 ) as dashed lines. 
Table 2

Experimental details.

\begin{tabular}{|c|c|}
\hline \multicolumn{2}{|l|}{ Crystal data } \\
\hline Chemical formula & $\mathrm{C}_{22} \mathrm{H}_{31} \mathrm{~N}_{3} \mathrm{O}_{6} \mathrm{~S}_{2}$ \\
\hline$M_{\mathrm{r}}$ & 497.62 \\
\hline Crystal system, space group & Triclinic, $P 1$ \\
\hline Temperature $(\mathrm{K})$ & 296 \\
\hline$a, b, c(\AA)$ & $\begin{array}{l}7.7292(10), 7.9892(9) \\
11.2035(13)\end{array}$ \\
\hline$\alpha, \beta, \gamma\left({ }^{\circ}\right)$ & $108.300(7), 92.553(7), 101.499(8)$ \\
\hline$V\left(\AA^{3}\right)$ & $639.36(14)$ \\
\hline$Z$ & 1 \\
\hline Radiation type & $\mathrm{Cu} K \alpha$ \\
\hline$\mu\left(\mathrm{mm}^{-1}\right)$ & 2.23 \\
\hline Crystal size $(\mathrm{mm})$ & $0.17 \times 0.12 \times 0.10$ \\
\hline \multicolumn{2}{|l|}{ Data collection } \\
\hline Diffractometer & Bruker APEXII CCD \\
\hline Absorption correction & $\begin{array}{l}\text { Multi-scan (SADABS; Bruker, } \\
\text { 2014) }\end{array}$ \\
\hline$T_{\min }, T_{\max }$ & $0.703,0.808$ \\
\hline $\begin{array}{l}\text { No. of measured, independent and } \\
\text { observed }[I>2 \sigma(I)] \text { reflections }\end{array}$ & $3454,2483,2389$ \\
\hline$R_{\text {int }}$ & 0.019 \\
\hline$(\sin \theta / \lambda)_{\max }\left(\AA^{-1}\right)$ & 0.592 \\
\hline \multicolumn{2}{|l|}{ Refinement } \\
\hline$R\left[F^{2}>2 \sigma\left(F^{2}\right)\right], w R\left(F^{2}\right), S$ & $0.042,0.115,1.04$ \\
\hline No. of reflections & 2483 \\
\hline No. of parameters & 298 \\
\hline No. of restraints & 3 \\
\hline $\mathrm{H}$-atom treatment & $\mathrm{H}$-atom parameters constrained \\
\hline$\Delta \rho_{\max }, \Delta \rho_{\min }\left(\mathrm{e} \AA^{-3}\right)$ & $0.33,-0.21$ \\
\hline Absolute structure & $\begin{array}{l}\text { Flack } x \text { determined using } 531 \\
\quad \text { quotients }\left[\left(I^{+}\right)-\left(I^{-}\right)\right] /\left[\left(I^{+}\right)+\left(I^{-}\right)\right] \\
\text {(Parsons } \text { et al., 2013) }\end{array}$ \\
\hline Absolute structure parameter & $0.140(12)$ \\
\hline
\end{tabular}

Computer programs: APEX2 and SAINT (Bruker, 2014), SHELXS2018/3 and SHELXTL (Sheldrick, 2008) and SHELXL2018/3 (Sheldrick, 2015).

of using column chromatography, we successfully obtained pure tebipenem pivoxil on a relatively large scale through recrystallization from ethyl acetate, yielding colourless blockshaped crystals. The HPLC spectrum of the final product showed a single peak with less than $0.1 \%$ of impurities. $[\alpha]_{\mathrm{D}}{ }^{8}=$ $+9.6^{\circ}$, m.p. $=407-409 \mathrm{~K}$. Elemental analysis calculated for $\mathrm{C}_{22} \mathrm{H}_{31} \mathrm{~N}_{3} \mathrm{O}_{6} \mathrm{~S}_{2}$ : C, 53.10; H, 6.28; N, 8.44; S, 12.89; Found: C, 53.13; H, 6.32; N, 8.45; S, 12.94. HRESI-MS calculated for $\mathrm{C}_{22} \mathrm{H}_{32} \mathrm{~N}_{3} \mathrm{O}_{6} \mathrm{~S}_{2}\left([M+\mathrm{H}]^{+}\right): 498.1727$, found: 498.1867. The structure has also been characterized with ${ }^{1} \mathrm{H}$ NMR, ${ }^{13} \mathrm{C}$ NMR, and IR spectroscopy. ${ }^{1} \mathrm{H}$ NMR, ${ }^{13} \mathrm{C} \mathrm{NMR}$, and IR spectra of tebipenem pivoxil $\mathbf{1}$ are included in the supporting information and compared with reference values, including the assignment of NMR chemical shifts and IR absorption bands (Isoda et al., 2006a).

\section{Refinement}

Crystal data, data collection and structure refinement details are summarized in Table 2. In the refinement, all $\mathrm{H}$ atoms were positioned geometrically and refined as riding: $\mathrm{C}-\mathrm{H}=$ $0.96-0.98 \AA$ with $U_{\text {iso }}(\mathrm{H})=1.2 U_{\text {eq }}(\mathrm{C})$ or $1.5 U_{\text {eq }}(\mathrm{C}$-methyl $)$.

\section{Acknowledgements}

The authors thank Dr Jialiang Zhong at the China State Institute of Pharmaceutical Industry for helpful discussion.

\section{References}

Bruker (2014). APEX2, SAINT and SADABS. Bruker AXS Inc., Madison Wisconsin, USA.

Groom, C. R., Bruno, I. J., Lightfoot, M. P. \& Ward, S. C. (2016). Acta Cryst. B72, 171-179.

Isoda, T., Ushirogochi, H., Satoh, K., Takasaki, T., Yamamura, I., Sato, C., Mihira, A., Abe, T., Tamai, S., Yamamoto, S., Kumagai, T. \& Nagao, Y. (2006a). J. Antibiot. 59, 241-247.

Isoda, T., Yamamura, I., Tamai, S., Kumagai, T. \& Nagao, Y. (2006b). Chem. Pharm. Bull. 54, 1408-1411.

Johnston, D. B. R., Schmitt, S. M., Bouffard, F. A. \& Christensen, B. G. (1978). J. Am. Chem. Soc. 100, 313-315.

Kato, K., Shirasaka, Y., Kuraoka, E., Kikuchi, A., Iguchi, M., Suzuki, H., Shibasaki, S., Kurosawa, T. \& Tamai, I. (2010). Mol. Pharm. 7, 1747-1756.

Kijima, K., Morita, J., Suzuki, K., Aoki, M., Kato, K., Hayashi, H., Shibasaki, S. \& Kurosawa, T. (2009). Jpn. J. Antibiot. 62, 214-240.

Kobayashi, R., Konomi, M., Hasegawa, K., Morozumi, M., Sunakawa, K. \& Ubukata, K. (2005). Antimicrob. Agents Chemother. 49, 889894.

Papp-Wallace, K. M., Endimiani, A., Taracila, M. A. \& Bonomo, R. A. (2011). Antimicrob. Agents Chemother. 55, 4943-4960.

Parsons, S., Flack, H. D. \& Wagner, T. (2013). Acta Cryst. B69, 249 259.

Sheldrick, G. M. (2008). Acta Cryst. A64, 112-122.

Sheldrick, G. M. (2015). Acta Cryst. C71, 3-8. 


\section{supporting information}

Acta Cryst. (2018). E74, 1215-1217 [https://doi.org/10.1107/S2056989018010770]

\section{Crystal structure of tebipenem pivoxil}

\section{Chao Tang, Li Cai, Shuai Liu, Zhiwei Zheng, Gen Li, Jianli Chen and Qiang Sui}

\section{Computing details}

Data collection: APEX2 (Bruker, 2014); cell refinement: SAINT (Bruker, 2014); data reduction: SAINT (Bruker, 2014); program(s) used to solve structure: SHELXS2018/3 (Sheldrick, 2008); program(s) used to refine structure:

SHELXL2018/3 (Sheldrick, 2015); molecular graphics: SHELXTL (Sheldrick, 2008); software used to prepare material for publication: SHELXTL (Sheldrick, 2008).

$\backslash$ (2,2-Dimethylpropanoyloxy)methyl (4R,5S,6S)-3-\{[1-(4,5-dihydro-1,3-thiazol-2-yl)azetidin- $\backslash 3$ yl]sulfanyl\}-6-[(1R)-1-hydroxyethyl]-4-methyl-7-oxo-1- \azabicyclo[3.2.0]hept-2-ene-2-carboxylate

Crystal data

$\mathrm{C}_{22} \mathrm{H}_{31} \mathrm{~N}_{3} \mathrm{O}_{6} \mathrm{~S}_{2}$

$M_{r}=497.62$

Triclinic, $P 1$

$a=7.7292(10) \AA$

$b=7.9892(9) \AA$

$c=11.2035(13) \AA$

$\alpha=108.300(7)^{\circ}$

$\beta=92.553(7)^{\circ}$

$\gamma=101.499(8)^{\circ}$

$V=639.36(14) \AA^{3}$

Data collection

Bruker APEXII CCD

diffractometer

$\varphi$ and $\omega$ scans

Absorption correction: multi-scan

(SADABS; Bruker, 2014)

$T_{\min }=0.703, T_{\max }=0.808$

3454 measured reflections

\section{Refinement}

Refinement on $F^{2}$

Least-squares matrix: full

$R\left[F^{2}>2 \sigma\left(F^{2}\right)\right]=0.042$

$w R\left(F^{2}\right)=0.115$

$S=1.04$

2483 reflections

298 parameters

3 restraints

Hydrogen site location: inferred from neighbouring sites
$Z=1$

$F(000)=264$

$D_{\mathrm{x}}=1.292 \mathrm{Mg} \mathrm{m}^{-3}$

$\mathrm{Cu} K \alpha$ radiation, $\lambda=1.54178 \AA$

Cell parameters from 2598 reflections

$\theta=4.2-65.6^{\circ}$

$\mu=2.23 \mathrm{~mm}^{-1}$

$T=296 \mathrm{~K}$

Block, colorless

$0.17 \times 0.12 \times 0.10 \mathrm{~mm}$

2483 independent reflections

2389 reflections with $I>2 \sigma(I)$

$R_{\text {int }}=0.019$

$\theta_{\max }=65.9^{\circ}, \theta_{\min }=4.2^{\circ}$

$h=-7 \rightarrow 9$

$k=-9 \rightarrow 9$

$l=-13 \rightarrow 12$

$\mathrm{H}$-atom parameters constrained

$w=1 /\left[\sigma^{2}\left(F_{\mathrm{o}}^{2}\right)+(0.0826 P)^{2}+0.0878 P\right]$

where $P=\left(F_{\mathrm{o}}{ }^{2}+2 F_{\mathrm{c}}{ }^{2}\right) / 3$

$(\Delta / \sigma)_{\max }=0.015$

$\Delta \rho_{\max }=0.33 \mathrm{e} \AA^{-3}$

$\Delta \rho_{\min }=-0.21$ e $\AA^{-3}$

Absolute structure: Flack $x$ determined using

531 quotients $\left[\left(I^{+}\right)-(I)\right] /\left[\left(I^{+}\right)+\left(I^{-}\right)\right]$(Parsons et al., 2013)

Absolute structure parameter: 0.140 (12) 


\section{Special details}

Geometry. All esds (except the esd in the dihedral angle between two 1.s. planes) are estimated using the full covariance matrix. The cell esds are taken into account individually in the estimation of esds in distances, angles and torsion angles; correlations between esds in cell parameters are only used when they are defined by crystal symmetry. An approximate (isotropic) treatment of cell esds is used for estimating esds involving l.s. planes.

Fractional atomic coordinates and isotropic or equivalent isotropic displacement parameters $\left(\hat{A}^{2}\right)$

\begin{tabular}{|c|c|c|c|c|}
\hline & $x$ & $y$ & $z$ & $U_{\text {iso }} * / U_{\text {eq }}$ \\
\hline $\mathrm{S} 1$ & $0.33764(12)$ & $0.37913(11)$ & $0.01567(9)$ & $0.0531(3)$ \\
\hline $\mathrm{S} 2$ & 0.6657 (3) & $0.6243(2)$ & $0.50488(14)$ & $0.0889(5)$ \\
\hline $\mathrm{O} 1$ & $-0.0384(5)$ & $0.8665(5)$ & $-0.1269(3)$ & $0.0695(9)$ \\
\hline $\mathrm{O} 2$ & $-0.0964(4)$ & $1.0714(4)$ & $0.1973(3)$ & $0.0601(8)$ \\
\hline $\mathrm{H} 2 \mathrm{~A}$ & -0.139807 & 1.152553 & 0.240566 & $0.090^{*}$ \\
\hline $\mathrm{O} 3$ & 0.4728 (4) & $0.6066(5)$ & $-0.1353(3)$ & $0.0626(8)$ \\
\hline $\mathrm{O} 4$ & 0.3985 (4) & $0.8733(4)$ & $-0.1081(3)$ & $0.0589(8)$ \\
\hline O5 & $0.4493(5)$ & $1.0081(6)$ & $-0.2577(4)$ & $0.0781(11)$ \\
\hline O6 & $0.2673(7)$ & $0.7659(6)$ & $-0.3881(6)$ & $0.1060(16)$ \\
\hline N1 & $0.1226(4)$ & $0.7928(4)$ & $0.0285(3)$ & $0.0447(7)$ \\
\hline $\mathrm{N} 2$ & $0.5213(7)$ & $0.3896(6)$ & 0.2801 (4) & $0.0737(12)$ \\
\hline N3 & $0.7629(6)$ & $0.3330(6)$ & 0.3725 (4) & $0.0679(11)$ \\
\hline $\mathrm{C} 1$ & $-0.0250(6)$ & $0.8214(6)$ & $-0.0352(4)$ & $0.0486(9)$ \\
\hline $\mathrm{C} 2$ & $-0.1483(5)$ & $0.7724(5)$ & $0.0574(4)$ & $0.0455(8)$ \\
\hline $\mathrm{H} 2 \mathrm{~B}$ & -0.238265 & 0.661201 & 0.014328 & $0.055^{*}$ \\
\hline $\mathrm{C} 3$ & $0.0144(5)$ & $0.7296(5)$ & 0.1179 (4) & $0.0442(8)$ \\
\hline H3B & 0.050788 & 0.808606 & 0.205772 & $0.053^{*}$ \\
\hline $\mathrm{C} 4$ & 0.0397 (6) & $0.5350(6)$ & 0.0968 (4) & $0.0498(9)$ \\
\hline $\mathrm{H} 4 \mathrm{~A}$ & 0.058878 & 0.516830 & 0.178516 & $0.060^{*}$ \\
\hline $\mathrm{C} 5$ & $0.2114(5)$ & $0.5408(5)$ & $0.0341(4)$ & $0.0437(8)$ \\
\hline C6 & $0.2416(5)$ & $0.6785(5)$ & $-0.0123(4)$ & $0.0436(8)$ \\
\hline $\mathrm{C} 7$ & $-0.2320(5)$ & $0.9153(5)$ & $0.1391(4)$ & $0.0483(9)$ \\
\hline $\mathrm{H} 7 \mathrm{~A}$ & -0.316888 & 0.944012 & 0.085209 & $0.058^{*}$ \\
\hline $\mathrm{C} 8$ & $-0.3299(7)$ & $0.8508(7)$ & $0.2361(5)$ & $0.0651(12)$ \\
\hline $\mathrm{H} 8 \mathrm{~A}$ & -0.421147 & 0.744567 & 0.193571 & $0.098^{*}$ \\
\hline H8B & -0.247930 & 0.822482 & 0.289674 & $0.098^{*}$ \\
\hline $\mathrm{H} 8 \mathrm{C}$ & -0.382809 & 0.944259 & 0.286473 & $0.098^{*}$ \\
\hline $\mathrm{C} 9$ & $-0.1081(6)$ & $0.3822(7)$ & $0.0108(7)$ & $0.0762(16)$ \\
\hline H9A & -0.215396 & 0.379378 & 0.050815 & $0.114^{*}$ \\
\hline H9B & -0.127753 & 0.402322 & -0.068287 & $0.114^{*}$ \\
\hline $\mathrm{H} 9 \mathrm{C}$ & -0.073970 & 0.268830 & -0.004165 & $0.114 *$ \\
\hline $\mathrm{C} 10$ & $0.3811(5)$ & 0.7099 (6) & $-0.0923(4)$ & $0.0472(9)$ \\
\hline C11 & $0.2935(6)$ & $0.3012(6)$ & $0.1482(5)$ & $0.0535(10)$ \\
\hline H11A & 0.175564 & 0.222769 & 0.139734 & $0.064 *$ \\
\hline C12 & $0.4501(6)$ & $0.2200(5)$ & $0.1758(5)$ & $0.0532(10)$ \\
\hline $\mathrm{H} 12 \mathrm{~A}$ & 0.415244 & 0.115292 & 0.202918 & $0.064 *$ \\
\hline H12B & 0.525236 & 0.196247 & 0.108146 & $0.064 *$ \\
\hline C13 & $0.3520(6)$ & $0.4482(6)$ & $0.2791(5)$ & $0.0581(11)$ \\
\hline H13A & 0.364717 & 0.570857 & 0.277752 & $0.070^{*}$ \\
\hline
\end{tabular}




$\begin{array}{lllll}\text { H13B } & 0.280849 & 0.428426 & 0.344663 & 0.070^{*} \\ \text { C14 } & 0.6465(6) & 0.4232(6) & 0.3738(4) & 0.0559(11) \\ \text { C15 } & 0.8971(11) & 0.4207(12) & 0.4850(8) & 0.116(3) \\ \text { H15A } & 1.004355 & 0.482093 & 0.461013 & 0.140^{*} \\ \text { H15B } & 0.927124 & 0.329086 & 0.516839 & 0.140^{*} \\ \text { C16 } & 0.8293(11) & 0.5528(11) & 0.5860(6) & 0.098(2) \\ \text { H16A } & 0.924735 & 0.655012 & 0.632517 & 0.118^{*} \\ \text { H16B } & 0.775573 & 0.496738 & 0.644719 & 0.118^{*} \\ \text { C17 } & 0.5186(7) & 0.9092(9) & -0.1956(6) & 0.0739(14) \\ \text { H17A } & 0.634318 & 0.976545 & -0.150543 & 0.089^{*} \\ \text { H17B } & 0.532612 & 0.796482 & -0.255647 & 0.089^{*} \\ \text { C18 } & 0.3224(8) & 0.9266(8) & -0.3535(5) & 0.0724(14) \\ \text { C19 } & 0.2625(11) & 1.0577(9) & -0.4111(5) & 0.0854(18) \\ \text { C20 } & 0.406(2) & 1.2163(17) & -0.4024(14) & 0.181(6) \\ \text { H20A } & 0.455241 & 1.276802 & -0.315584 & 0.271^{*} \\ \text { H20B } & 0.497695 & 1.176572 & -0.451723 & 0.271^{*} \\ \text { H20C } & 0.358495 & 1.298285 & -0.434103 & 0.271^{*} \\ \text { C21 } & 0.179(2) & 0.9556(19) & -0.5419(9) & 0.183(6) \\ \text { H21A } & 0.087040 & 0.855032 & -0.541445 & 0.274^{*} \\ \text { H21B } & 0.127395 & 1.033571 & -0.575774 & 0.274^{*} \\ \text { H21C } & 0.266596 & 0.911858 & -0.593394 & 0.274^{*} \\ \text { C22 } & 0.1304(18) & 1.1339(17) & -0.3284(9) & 0.142(4) \\ \text { H22A } & 0.188241 & 1.199331 & -0.244299 & 0.213^{*} \\ \text { H22B } & 0.082558 & 1.214142 & -0.361897 & 0.213^{*} \\ \text { H22C } & 0.035952 & 1.036984 & -0.326218 & 0.213^{*} \\ & & & & \end{array}$

Atomic displacement parameters $\left(\AA^{2}\right)$

\begin{tabular}{lllllll}
\hline & $U^{11}$ & $U^{22}$ & $U^{33}$ & $U^{12}$ & $U^{13}$ & $U^{23}$ \\
\hline S1 & $0.0556(6)$ & $0.0528(5)$ & $0.0564(6)$ & $0.0265(4)$ & $0.0067(4)$ & $0.0168(4)$ \\
S2 & $0.1153(13)$ & $0.0862(10)$ & $0.0592(7)$ & $0.0530(9)$ & $0.0044(7)$ & $-0.0025(7)$ \\
O1 & $0.078(2)$ & $0.096(3)$ & $0.0542(19)$ & $0.0412(19)$ & $0.0077(16)$ & $0.0382(18)$ \\
O2 & $0.0553(17)$ & $0.0497(16)$ & $0.070(2)$ & $0.0134(14)$ & $0.0171(15)$ & $0.0107(15)$ \\
O3 & $0.0572(18)$ & $0.0701(19)$ & $0.067(2)$ & $0.0282(17)$ & $0.0193(15)$ & $0.0220(16)$ \\
O4 & $0.0610(18)$ & $0.0706(18)$ & $0.0592(18)$ & $0.0253(15)$ & $0.0183(14)$ & $0.0332(16)$ \\
O5 & $0.081(2)$ & $0.102(3)$ & $0.0598(19)$ & $0.014(2)$ & $0.0054(17)$ & $0.042(2)$ \\
O6 & $0.099(3)$ & $0.077(3)$ & $0.126(4)$ & $0.020(2)$ & $-0.015(3)$ & $0.014(3)$ \\
N1 & $0.0461(17)$ & $0.0475(17)$ & $0.0448(17)$ & $0.0201(14)$ & $0.0055(14)$ & $0.0155(14)$ \\
N2 & $0.072(3)$ & $0.076(3)$ & $0.068(3)$ & $0.045(2)$ & $-0.005(2)$ & $0.002(2)$ \\
N3 & $0.064(2)$ & $0.065(2)$ & $0.064(2)$ & $0.029(2)$ & $-0.0105(19)$ & $0.001(2)$ \\
C1 & $0.050(2)$ & $0.052(2)$ & $0.041(2)$ & $0.0208(18)$ & $0.0003(16)$ & $0.0081(18)$ \\
C2 & $0.0405(19)$ & $0.048(2)$ & $0.046(2)$ & $0.0151(16)$ & $-0.0009(16)$ & $0.0099(17)$ \\
C3 & $0.043(2)$ & $0.052(2)$ & $0.0400(18)$ & $0.0173(16)$ & $0.0043(15)$ & $0.0141(17)$ \\
C4 & $0.044(2)$ & $0.058(2)$ & $0.058(2)$ & $0.0244(18)$ & $0.0084(17)$ & $0.026(2)$ \\
C5 & $0.0399(18)$ & $0.0476(19)$ & $0.0424(19)$ & $0.0142(16)$ & $-0.0011(15)$ & $0.0112(17)$ \\
C6 & $0.0399(19)$ & $0.047(2)$ & $0.0416(18)$ & $0.0131(16)$ & $0.0002(14)$ & $0.0097(16)$ \\
C7 & $0.0414(19)$ & $0.048(2)$ & $0.058(2)$ & $0.0179(17)$ & $0.0050(17)$ & $0.0158(18)$ \\
C8 & $0.062(3)$ & $0.061(3)$ & $0.079(3)$ & $0.019(2)$ & $0.024(2)$ & $0.026(2)$
\end{tabular}


supporting information

\begin{tabular}{lllllll}
\hline C9 & $0.045(2)$ & $0.053(3)$ & $0.129(5)$ & $0.008(2)$ & $0.004(3)$ & $0.031(3)$ \\
C10 & $0.0422(19)$ & $0.057(2)$ & $0.043(2)$ & $0.0152(19)$ & $-0.0019(16)$ & $0.0155(18)$ \\
C11 & $0.045(2)$ & $0.051(2)$ & $0.070(3)$ & $0.0151(18)$ & $0.0032(19)$ & $0.026(2)$ \\
C12 & $0.057(2)$ & $0.043(2)$ & $0.062(2)$ & $0.0210(18)$ & $0.001(2)$ & $0.0162(18)$ \\
C13 & $0.059(3)$ & $0.066(3)$ & $0.063(3)$ & $0.035(2)$ & $0.016(2)$ & $0.026(2)$ \\
C14 & $0.063(3)$ & $0.057(2)$ & $0.049(2)$ & $0.025(2)$ & $0.008(2)$ & $0.013(2)$ \\
C15 & $0.098(5)$ & $0.119(6)$ & $0.102(5)$ & $0.055(5)$ & $-0.043(5)$ & $-0.016(5)$ \\
C16 & $0.101(5)$ & $0.115(5)$ & $0.067(4)$ & $0.036(4)$ & $-0.014(3)$ & $0.010(4)$ \\
C17 & $0.056(3)$ & $0.113(4)$ & $0.070(3)$ & $0.024(3)$ & $0.019(2)$ & $0.051(3)$ \\
C18 & $0.069(3)$ & $0.072(3)$ & $0.069(3)$ & $0.012(3)$ & $0.008(3)$ & $0.016(3)$ \\
C19 & $0.128(5)$ & $0.087(4)$ & $0.048(3)$ & $0.037(4)$ & $0.001(3)$ & $0.025(3)$ \\
C20 & $0.247(16)$ & $0.137(9)$ & $0.167(11)$ & $0.007(9)$ & $-0.005(11)$ & $0.089(8)$ \\
C21 & $0.267(18)$ & $0.196(12)$ & $0.070(5)$ & $0.079(12)$ & $-0.037(7)$ & $0.014(6)$ \\
C22 & $0.190(11)$ & $0.185(10)$ & $0.095(6)$ & $0.122(9)$ & $0.017(6)$ & $0.057(6)$ \\
& & & & & & \\
\hline
\end{tabular}

Geometric parameters $\left(A,{ }^{\circ}\right)$

\begin{tabular}{|c|c|c|c|}
\hline $\mathrm{S} 1-\mathrm{C} 5$ & $1.737(4)$ & $\mathrm{C} 8-\mathrm{H} 8 \mathrm{~A}$ & 0.9600 \\
\hline $\mathrm{S} 1-\mathrm{C} 11$ & $1.802(4)$ & $\mathrm{C} 8-\mathrm{H} 8 \mathrm{~B}$ & 0.9600 \\
\hline $\mathrm{S} 2-\mathrm{C} 14$ & $1.778(5)$ & $\mathrm{C} 8-\mathrm{H} 8 \mathrm{C}$ & 0.9600 \\
\hline $\mathrm{S} 2-\mathrm{C} 16$ & $1.807(7)$ & C9-H9A & 0.9600 \\
\hline $\mathrm{O} 1-\mathrm{C} 1$ & $1.197(5)$ & $\mathrm{C} 9-\mathrm{H} 9 \mathrm{~B}$ & 0.9600 \\
\hline $\mathrm{O} 2-\mathrm{C} 7$ & 1.409 (5) & $\mathrm{C} 9-\mathrm{H} 9 \mathrm{C}$ & 0.9600 \\
\hline $\mathrm{O} 2-\mathrm{H} 2 \mathrm{~A}$ & 0.8200 & $\mathrm{C} 11-\mathrm{C} 13$ & $1.544(7)$ \\
\hline $\mathrm{O} 3-\mathrm{C} 10$ & $1.196(5)$ & $\mathrm{C} 11-\mathrm{C} 12$ & $1.546(6)$ \\
\hline $\mathrm{O} 4-\mathrm{C} 10$ & $1.354(5)$ & C11-H11A & 0.9800 \\
\hline $\mathrm{O} 4-\mathrm{C} 17$ & $1.433(6)$ & $\mathrm{C} 12-\mathrm{H} 12 \mathrm{~A}$ & 0.9700 \\
\hline $\mathrm{O} 5-\mathrm{C} 18$ & $1.323(7)$ & $\mathrm{C} 12-\mathrm{H} 12 \mathrm{~B}$ & 0.9700 \\
\hline $\mathrm{O} 5-\mathrm{C} 17$ & $1.370(7)$ & $\mathrm{C} 13-\mathrm{H} 13 \mathrm{~A}$ & 0.9700 \\
\hline $\mathrm{O} 6-\mathrm{C} 18$ & $1.198(7)$ & C13-H13B & 0.9700 \\
\hline $\mathrm{N} 1-\mathrm{C} 1$ & $1.413(5)$ & $\mathrm{C} 15-\mathrm{C} 16$ & $1.480(11)$ \\
\hline $\mathrm{N} 1-\mathrm{C} 6$ & $1.414(6)$ & $\mathrm{C} 15-\mathrm{H} 15 \mathrm{~A}$ & 0.9700 \\
\hline $\mathrm{N} 1-\mathrm{C} 3$ & $1.477(5)$ & C15-H15B & 0.9700 \\
\hline $\mathrm{N} 2-\mathrm{C} 14$ & $1.319(6)$ & $\mathrm{C} 16-\mathrm{H} 16 \mathrm{~A}$ & 0.9700 \\
\hline $\mathrm{N} 2-\mathrm{C} 12$ & $1.468(6)$ & C16-H16B & 0.9700 \\
\hline $\mathrm{N} 2-\mathrm{C} 13$ & $1.476(6)$ & C17-H17A & 0.9700 \\
\hline $\mathrm{N} 3-\mathrm{C} 14$ & $1.258(6)$ & C17-H17B & 0.9700 \\
\hline $\mathrm{N} 3-\mathrm{C} 15$ & $1.481(7)$ & $\mathrm{C} 18-\mathrm{C} 19$ & $1.523(8)$ \\
\hline $\mathrm{C} 1-\mathrm{C} 2$ & $1.527(6)$ & $\mathrm{C} 19-\mathrm{C} 21$ & $1.479(10)$ \\
\hline $\mathrm{C} 2-\mathrm{C} 7$ & $1.500(6)$ & $\mathrm{C} 19-\mathrm{C} 20$ & $1.483(15)$ \\
\hline $\mathrm{C} 2-\mathrm{C} 3$ & $1.549(5)$ & $\mathrm{C} 19-\mathrm{C} 22$ & $1.499(13)$ \\
\hline $\mathrm{C} 2-\mathrm{H} 2 \mathrm{~B}$ & 0.9800 & $\mathrm{C} 20-\mathrm{H} 20 \mathrm{~A}$ & 0.9600 \\
\hline $\mathrm{C} 3-\mathrm{C} 4$ & $1.551(6)$ & $\mathrm{C} 20-\mathrm{H} 20 \mathrm{~B}$ & 0.9600 \\
\hline $\mathrm{C} 3-\mathrm{H} 3 \mathrm{~B}$ & 0.9800 & $\mathrm{C} 20-\mathrm{H} 20 \mathrm{C}$ & 0.9600 \\
\hline $\mathrm{C} 4-\mathrm{C} 9$ & $1.527(7)$ & $\mathrm{C} 21-\mathrm{H} 21 \mathrm{~A}$ & 0.9600 \\
\hline $\mathrm{C} 4-\mathrm{C} 5$ & $1.528(6)$ & $\mathrm{C} 21-\mathrm{H} 21 \mathrm{~B}$ & 0.9600 \\
\hline $\mathrm{C} 4-\mathrm{H} 4 \mathrm{~A}$ & 0.9800 & $\mathrm{C} 21-\mathrm{H} 21 \mathrm{C}$ & 0.9600 \\
\hline $\mathrm{C} 5-\mathrm{C} 6$ & $1.342(6)$ & $\mathrm{C} 22-\mathrm{H} 22 \mathrm{~A}$ & 0.9600 \\
\hline
\end{tabular}




\begin{tabular}{|c|c|c|c|}
\hline $\mathrm{C} 6-\mathrm{C} 10$ & $1.466(6)$ & $\mathrm{C} 22-\mathrm{H} 22 \mathrm{~B}$ & 0.9600 \\
\hline $\mathrm{C} 7-\mathrm{C} 8$ & $1.512(6)$ & $\mathrm{C} 22-\mathrm{H} 22 \mathrm{C}$ & 0.9600 \\
\hline $\mathrm{C} 7-\mathrm{H} 7 \mathrm{~A}$ & 0.9800 & & \\
\hline $\mathrm{C} 5-\mathrm{S} 1-\mathrm{C} 11$ & $102.76(19)$ & $\mathrm{C} 13-\mathrm{C} 11-\mathrm{H} 11 \mathrm{~A}$ & 114.7 \\
\hline $\mathrm{C} 14-\mathrm{S} 2-\mathrm{C} 16$ & $88.8(3)$ & $\mathrm{C} 12-\mathrm{C} 11-\mathrm{H} 11 \mathrm{~A}$ & 114.7 \\
\hline $\mathrm{C} 7-\mathrm{O} 2-\mathrm{H} 2 \mathrm{~A}$ & 109.5 & $\mathrm{~S} 1-\mathrm{C} 11-\mathrm{H} 11 \mathrm{~A}$ & 114.7 \\
\hline $\mathrm{C} 10-\mathrm{O} 4-\mathrm{C} 17$ & $116.1(4)$ & $\mathrm{N} 2-\mathrm{C} 12-\mathrm{C} 11$ & $88.2(3)$ \\
\hline $\mathrm{C} 18-\mathrm{O} 5-\mathrm{C} 17$ & $120.2(5)$ & $\mathrm{N} 2-\mathrm{C} 12-\mathrm{H} 12 \mathrm{~A}$ & 114.0 \\
\hline $\mathrm{C} 1-\mathrm{N} 1-\mathrm{C} 6$ & $132.4(3)$ & $\mathrm{C} 11-\mathrm{C} 12-\mathrm{H} 12 \mathrm{~A}$ & 114.0 \\
\hline $\mathrm{C} 1-\mathrm{N} 1-\mathrm{C} 3$ & $93.1(3)$ & $\mathrm{N} 2-\mathrm{C} 12-\mathrm{H} 12 \mathrm{~B}$ & 114.0 \\
\hline $\mathrm{C} 6-\mathrm{N} 1-\mathrm{C} 3$ & $108.6(3)$ & $\mathrm{C} 11-\mathrm{C} 12-\mathrm{H} 12 \mathrm{~B}$ & 114.0 \\
\hline $\mathrm{C} 14-\mathrm{N} 2-\mathrm{C} 12$ & $128.7(4)$ & $\mathrm{H} 12 \mathrm{~A}-\mathrm{C} 12-\mathrm{H} 12 \mathrm{~B}$ & 111.2 \\
\hline $\mathrm{C} 14-\mathrm{N} 2-\mathrm{C} 13$ & $130.5(5)$ & $\mathrm{N} 2-\mathrm{C} 13-\mathrm{C} 11$ & $88.0(4)$ \\
\hline $\mathrm{C} 12-\mathrm{N} 2-\mathrm{C} 13$ & $92.8(4)$ & $\mathrm{N} 2-\mathrm{C} 13-\mathrm{H} 13 \mathrm{~A}$ & 114.0 \\
\hline $\mathrm{C} 14-\mathrm{N} 3-\mathrm{C} 15$ & $111.1(5)$ & $\mathrm{C} 11-\mathrm{C} 13-\mathrm{H} 13 \mathrm{~A}$ & 114.0 \\
\hline $\mathrm{O} 1-\mathrm{C} 1-\mathrm{N} 1$ & $131.4(4)$ & $\mathrm{N} 2-\mathrm{C} 13-\mathrm{H} 13 \mathrm{~B}$ & 114.0 \\
\hline $\mathrm{O} 1-\mathrm{C} 1-\mathrm{C} 2$ & $136.6(4)$ & $\mathrm{C} 11-\mathrm{C} 13-\mathrm{H} 13 \mathrm{~B}$ & 114.0 \\
\hline $\mathrm{N} 1-\mathrm{C} 1-\mathrm{C} 2$ & $91.9(3)$ & $\mathrm{H} 13 \mathrm{~A}-\mathrm{C} 13-\mathrm{H} 13 \mathrm{~B}$ & 111.2 \\
\hline $\mathrm{C} 7-\mathrm{C} 2-\mathrm{C} 1$ & $118.6(3)$ & $\mathrm{N} 3-\mathrm{C} 14-\mathrm{N} 2$ & $124.9(5)$ \\
\hline $\mathrm{C} 7-\mathrm{C} 2-\mathrm{C} 3$ & $117.9(3)$ & $\mathrm{N} 3-\mathrm{C} 14-\mathrm{S} 2$ & $117.5(4)$ \\
\hline $\mathrm{C} 1-\mathrm{C} 2-\mathrm{C} 3$ & $86.1(3)$ & $\mathrm{N} 2-\mathrm{C} 14-\mathrm{S} 2$ & $117.3(4)$ \\
\hline $\mathrm{C} 7-\mathrm{C} 2-\mathrm{H} 2 \mathrm{~B}$ & 110.7 & $\mathrm{~N} 3-\mathrm{C} 15-\mathrm{C} 16$ & $111.0(6)$ \\
\hline $\mathrm{C} 1-\mathrm{C} 2-\mathrm{H} 2 \mathrm{~B}$ & 110.7 & $\mathrm{~N} 3-\mathrm{C} 15-\mathrm{H} 15 \mathrm{~A}$ & 109.4 \\
\hline $\mathrm{C} 3-\mathrm{C} 2-\mathrm{H} 2 \mathrm{~B}$ & 110.7 & $\mathrm{C} 16-\mathrm{C} 15-\mathrm{H} 15 \mathrm{~A}$ & 109.4 \\
\hline $\mathrm{N} 1-\mathrm{C} 3-\mathrm{C} 2$ & $88.6(3)$ & $\mathrm{N} 3-\mathrm{C} 15-\mathrm{H} 15 \mathrm{~B}$ & 109.4 \\
\hline $\mathrm{N} 1-\mathrm{C} 3-\mathrm{C} 4$ & $104.6(3)$ & $\mathrm{C} 16-\mathrm{C} 15-\mathrm{H} 15 \mathrm{~B}$ & 109.4 \\
\hline $\mathrm{C} 2-\mathrm{C} 3-\mathrm{C} 4$ & $123.7(3)$ & $\mathrm{H} 15 \mathrm{~A}-\mathrm{C} 15-\mathrm{H} 15 \mathrm{~B}$ & 108.0 \\
\hline $\mathrm{N} 1-\mathrm{C} 3-\mathrm{H} 3 \mathrm{~B}$ & 112.2 & $\mathrm{C} 15-\mathrm{C} 16-\mathrm{S} 2$ & $105.3(5)$ \\
\hline $\mathrm{C} 2-\mathrm{C} 3-\mathrm{H} 3 \mathrm{~B}$ & 112.2 & $\mathrm{C} 15-\mathrm{C} 16-\mathrm{H} 16 \mathrm{~A}$ & 110.7 \\
\hline $\mathrm{C} 4-\mathrm{C} 3-\mathrm{H} 3 \mathrm{~B}$ & 112.2 & $\mathrm{~S} 2-\mathrm{C} 16-\mathrm{H} 16 \mathrm{~A}$ & 110.7 \\
\hline $\mathrm{C} 9-\mathrm{C} 4-\mathrm{C} 5$ & $109.9(4)$ & $\mathrm{C} 15-\mathrm{C} 16-\mathrm{H} 16 \mathrm{~B}$ & 110.7 \\
\hline $\mathrm{C} 9-\mathrm{C} 4-\mathrm{C} 3$ & $115.8(4)$ & $\mathrm{S} 2-\mathrm{C} 16-\mathrm{H} 16 \mathrm{~B}$ & 110.7 \\
\hline $\mathrm{C} 5-\mathrm{C} 4-\mathrm{C} 3$ & $100.8(3)$ & $\mathrm{H} 16 \mathrm{~A}-\mathrm{C} 16-\mathrm{H} 16 \mathrm{~B}$ & 108.8 \\
\hline $\mathrm{C} 9-\mathrm{C} 4-\mathrm{H} 4 \mathrm{~A}$ & 110.0 & $\mathrm{O} 5-\mathrm{C} 17-\mathrm{O} 4$ & $108.0(4)$ \\
\hline $\mathrm{C} 5-\mathrm{C} 4-\mathrm{H} 4 \mathrm{~A}$ & 110.0 & $\mathrm{O} 5-\mathrm{C} 17-\mathrm{H} 17 \mathrm{~A}$ & 110.1 \\
\hline $\mathrm{C} 3-\mathrm{C} 4-\mathrm{H} 4 \mathrm{~A}$ & 110.0 & $\mathrm{O} 4-\mathrm{C} 17-\mathrm{H} 17 \mathrm{~A}$ & 110.1 \\
\hline $\mathrm{C} 6-\mathrm{C} 5-\mathrm{C} 4$ & $110.6(4)$ & $\mathrm{O} 5-\mathrm{C} 17-\mathrm{H} 17 \mathrm{~B}$ & 110.1 \\
\hline $\mathrm{C} 6-\mathrm{C} 5-\mathrm{S} 1$ & $125.5(3)$ & $\mathrm{O} 4-\mathrm{C} 17-\mathrm{H} 17 \mathrm{~B}$ & 110.1 \\
\hline $\mathrm{C} 4-\mathrm{C} 5-\mathrm{S} 1$ & $123.7(3)$ & $\mathrm{H} 17 \mathrm{~A}-\mathrm{C} 17-\mathrm{H} 17 \mathrm{~B}$ & 108.4 \\
\hline $\mathrm{C} 5-\mathrm{C} 6-\mathrm{N} 1$ & $110.9(3)$ & $\mathrm{O} 6-\mathrm{C} 18-\mathrm{O} 5$ & $120.9(6)$ \\
\hline $\mathrm{C} 5-\mathrm{C} 6-\mathrm{C} 10$ & $125.1(4)$ & $\mathrm{O} 6-\mathrm{C} 18-\mathrm{C} 19$ & $126.3(6)$ \\
\hline $\mathrm{N} 1-\mathrm{C} 6-\mathrm{C} 10$ & $124.0(3)$ & $\mathrm{O} 5-\mathrm{C} 18-\mathrm{C} 19$ & $112.8(5)$ \\
\hline $\mathrm{O} 2-\mathrm{C} 7-\mathrm{C} 2$ & $107.8(3)$ & $\mathrm{C} 21-\mathrm{C} 19-\mathrm{C} 20$ & $113.4(9)$ \\
\hline $\mathrm{O} 2-\mathrm{C} 7-\mathrm{C} 8$ & $111.5(4)$ & $\mathrm{C} 21-\mathrm{C} 19-\mathrm{C} 22$ & $111.2(10)$ \\
\hline $\mathrm{C} 2-\mathrm{C} 7-\mathrm{C} 8$ & $111.3(4)$ & $\mathrm{C} 20-\mathrm{C} 19-\mathrm{C} 22$ & $105.0(9)$ \\
\hline $\mathrm{O} 2-\mathrm{C} 7-\mathrm{H} 7 \mathrm{~A}$ & 108.7 & $\mathrm{C} 21-\mathrm{C} 19-\mathrm{C} 18$ & $108.6(7)$ \\
\hline $\mathrm{C} 2-\mathrm{C} 7-\mathrm{H} 7 \mathrm{~A}$ & 108.7 & $\mathrm{C} 20-\mathrm{C} 19-\mathrm{C} 18$ & $113.3(8)$ \\
\hline
\end{tabular}




\begin{tabular}{|c|c|}
\hline $\mathrm{C} 8-\mathrm{C} 7-\mathrm{H} 7 \mathrm{~A}$ & 108.7 \\
\hline $\mathrm{C} 7-\mathrm{C} 8-\mathrm{H} 8 \mathrm{~A}$ & 109.5 \\
\hline $\mathrm{C} 7-\mathrm{C} 8-\mathrm{H} 8 \mathrm{~B}$ & 109.5 \\
\hline $\mathrm{H} 8 \mathrm{~A}-\mathrm{C} 8-\mathrm{H} 8 \mathrm{~B}$ & 109.5 \\
\hline $\mathrm{C} 7-\mathrm{C} 8-\mathrm{H} 8 \mathrm{C}$ & 109.5 \\
\hline $\mathrm{H} 8 \mathrm{~A}-\mathrm{C} 8-\mathrm{H} 8 \mathrm{C}$ & 109.5 \\
\hline $\mathrm{H} 8 \mathrm{~B}-\mathrm{C} 8-\mathrm{H} 8 \mathrm{C}$ & 109.5 \\
\hline $\mathrm{C} 4-\mathrm{C} 9-\mathrm{H} 9 \mathrm{~A}$ & 109.5 \\
\hline $\mathrm{C} 4-\mathrm{C} 9-\mathrm{H} 9 \mathrm{~B}$ & 109.5 \\
\hline $\mathrm{H} 9 \mathrm{~A}-\mathrm{C} 9-\mathrm{H} 9 \mathrm{~B}$ & 109.5 \\
\hline $\mathrm{C} 4-\mathrm{C} 9-\mathrm{H} 9 \mathrm{C}$ & 109.5 \\
\hline $\mathrm{H} 9 \mathrm{~A}-\mathrm{C} 9-\mathrm{H} 9 \mathrm{C}$ & 109.5 \\
\hline $\mathrm{H} 9 \mathrm{~B}-\mathrm{C} 9-\mathrm{H} 9 \mathrm{C}$ & 109.5 \\
\hline $\mathrm{O} 3-\mathrm{C} 10-\mathrm{O} 4$ & $123.8(4)$ \\
\hline $\mathrm{O} 3-\mathrm{C} 10-\mathrm{C} 6$ & $124.3(4)$ \\
\hline $\mathrm{O} 4-\mathrm{C} 10-\mathrm{C} 6$ & $111.9(4)$ \\
\hline $\mathrm{C} 13-\mathrm{C} 11-\mathrm{C} 12$ & $87.3(3)$ \\
\hline $\mathrm{C} 13-\mathrm{C} 11-\mathrm{S} 1$ & $114.7(3)$ \\
\hline $\mathrm{C} 12-\mathrm{C} 11-\mathrm{S} 1$ & $107.7(3)$ \\
\hline $\mathrm{C} 6-\mathrm{N} 1-\mathrm{C} 1-\mathrm{O} 1$ & $-56.9(7)$ \\
\hline $\mathrm{C} 3-\mathrm{N} 1-\mathrm{C} 1-\mathrm{O} 1$ & $-175.8(5)$ \\
\hline $\mathrm{C} 6-\mathrm{N} 1-\mathrm{C} 1-\mathrm{C} 2$ & $122.9(4)$ \\
\hline $\mathrm{C} 3-\mathrm{N} 1-\mathrm{C} 1-\mathrm{C} 2$ & $4.0(3)$ \\
\hline $\mathrm{O} 1-\mathrm{C} 1-\mathrm{C} 2-\mathrm{C} 7$ & $-64.2(7)$ \\
\hline $\mathrm{N} 1-\mathrm{C} 1-\mathrm{C} 2-\mathrm{C} 7$ & $116.0(4)$ \\
\hline $\mathrm{O} 1-\mathrm{C} 1-\mathrm{C} 2-\mathrm{C} 3$ & $176.0(6)$ \\
\hline $\mathrm{N} 1-\mathrm{C} 1-\mathrm{C} 2-\mathrm{C} 3$ & $-3.8(3)$ \\
\hline $\mathrm{C} 1-\mathrm{N} 1-\mathrm{C} 3-\mathrm{C} 2$ & $-3.9(3)$ \\
\hline $\mathrm{C} 6-\mathrm{N} 1-\mathrm{C} 3-\mathrm{C} 2$ & $-140.9(3)$ \\
\hline $\mathrm{C} 1-\mathrm{N} 1-\mathrm{C} 3-\mathrm{C} 4$ & $120.6(3)$ \\
\hline $\mathrm{C} 6-\mathrm{N} 1-\mathrm{C} 3-\mathrm{C} 4$ & $-16.3(4)$ \\
\hline $\mathrm{C} 7-\mathrm{C} 2-\mathrm{C} 3-\mathrm{N} 1$ & $-116.8(4)$ \\
\hline $\mathrm{C} 1-\mathrm{C} 2-\mathrm{C} 3-\mathrm{N} 1$ & $3.7(3)$ \\
\hline $\mathrm{C} 7-\mathrm{C} 2-\mathrm{C} 3-\mathrm{C} 4$ & $136.5(4)$ \\
\hline $\mathrm{C} 1-\mathrm{C} 2-\mathrm{C} 3-\mathrm{C} 4$ & $-103.1(4)$ \\
\hline $\mathrm{N} 1-\mathrm{C} 3-\mathrm{C} 4-\mathrm{C} 9$ & $-98.4(5)$ \\
\hline $\mathrm{C} 2-\mathrm{C} 3-\mathrm{C} 4-\mathrm{C} 9$ & $-0.1(6)$ \\
\hline $\mathrm{N} 1-\mathrm{C} 3-\mathrm{C} 4-\mathrm{C} 5$ & $20.2(4)$ \\
\hline $\mathrm{C} 2-\mathrm{C} 3-\mathrm{C} 4-\mathrm{C} 5$ & $118.5(4)$ \\
\hline $\mathrm{C} 9-\mathrm{C} 4-\mathrm{C} 5-\mathrm{C} 6$ & $104.1(4)$ \\
\hline $\mathrm{C} 3-\mathrm{C} 4-\mathrm{C} 5-\mathrm{C} 6$ & $-18.6(4)$ \\
\hline $\mathrm{C} 9-\mathrm{C} 4-\mathrm{C} 5-\mathrm{S} 1$ & $-71.2(5)$ \\
\hline $\mathrm{C} 3-\mathrm{C} 4-\mathrm{C} 5-\mathrm{S} 1$ & $166.1(3)$ \\
\hline $\mathrm{C} 11-\mathrm{S} 1-\mathrm{C} 5-\mathrm{C} 6$ & $156.3(3)$ \\
\hline $\mathrm{C} 11-\mathrm{S} 1-\mathrm{C} 5-\mathrm{C} 4$ & $-29.1(4)$ \\
\hline $\mathrm{C} 4-\mathrm{C} 5-\mathrm{C} 6-\mathrm{N} 1$ & $9.5(4)$ \\
\hline $\mathrm{S} 1-\mathrm{C} 5-\mathrm{C} 6-\mathrm{N} 1$ & $-175.3(3)$ \\
\hline
\end{tabular}

\begin{tabular}{|c|c|}
\hline $\mathrm{C} 22-\mathrm{C} 19-\mathrm{C} 18$ & $105.0(6)$ \\
\hline $\mathrm{C} 19-\mathrm{C} 20-\mathrm{H} 20 \mathrm{~A}$ & 109.5 \\
\hline $\mathrm{C} 19-\mathrm{C} 20-\mathrm{H} 20 \mathrm{~B}$ & 109.5 \\
\hline $\mathrm{H} 20 \mathrm{~A}-\mathrm{C} 20-\mathrm{H} 20 \mathrm{~B}$ & 109.5 \\
\hline $\mathrm{C} 19-\mathrm{C} 20-\mathrm{H} 20 \mathrm{C}$ & 109.5 \\
\hline $\mathrm{H} 20 \mathrm{~A}-\mathrm{C} 20-\mathrm{H} 20 \mathrm{C}$ & 109.5 \\
\hline $\mathrm{H} 20 \mathrm{~B}-\mathrm{C} 20-\mathrm{H} 20 \mathrm{C}$ & 109.5 \\
\hline $\mathrm{C} 19-\mathrm{C} 21-\mathrm{H} 21 \mathrm{~A}$ & 109.5 \\
\hline $\mathrm{C} 19-\mathrm{C} 21-\mathrm{H} 21 \mathrm{~B}$ & 109.5 \\
\hline $\mathrm{H} 21 \mathrm{~A}-\mathrm{C} 21-\mathrm{H} 21 \mathrm{~B}$ & 109.5 \\
\hline $\mathrm{C} 19-\mathrm{C} 21-\mathrm{H} 21 \mathrm{C}$ & 109.5 \\
\hline $\mathrm{H} 21 \mathrm{~A}-\mathrm{C} 21-\mathrm{H} 21 \mathrm{C}$ & 109.5 \\
\hline $\mathrm{H} 21 \mathrm{~B}-\mathrm{C} 21-\mathrm{H} 21 \mathrm{C}$ & 109.5 \\
\hline $\mathrm{C} 19-\mathrm{C} 22-\mathrm{H} 22 \mathrm{~A}$ & 109.5 \\
\hline $\mathrm{C} 19-\mathrm{C} 22-\mathrm{H} 22 \mathrm{~B}$ & 109.5 \\
\hline $\mathrm{H} 22 \mathrm{~A}-\mathrm{C} 22-\mathrm{H} 22 \mathrm{~B}$ & 109.5 \\
\hline $\mathrm{C} 19-\mathrm{C} 22-\mathrm{H} 22 \mathrm{C}$ & 109.5 \\
\hline $\mathrm{H} 22 \mathrm{~A}-\mathrm{C} 22-\mathrm{H} 22 \mathrm{C}$ & 109.5 \\
\hline $\mathrm{H} 22 \mathrm{~B}-\mathrm{C} 22-\mathrm{H} 22 \mathrm{C}$ & 109.5 \\
\hline $\mathrm{C} 17-\mathrm{O} 4-\mathrm{C} 10-\mathrm{O} 3$ & $8.5(6)$ \\
\hline $\mathrm{C} 17-\mathrm{O} 4-\mathrm{C} 10-\mathrm{C} 6$ & $-173.7(4)$ \\
\hline $\mathrm{C} 5-\mathrm{C} 6-\mathrm{C} 10-\mathrm{O} 3$ & $7.5(6)$ \\
\hline $\mathrm{N} 1-\mathrm{C} 6-\mathrm{C} 10-\mathrm{O} 3$ & $-173.8(4)$ \\
\hline $\mathrm{C} 5-\mathrm{C} 6-\mathrm{C} 10-\mathrm{O} 4$ & $-170.2(4)$ \\
\hline $\mathrm{N} 1-\mathrm{C} 6-\mathrm{C} 10-\mathrm{O} 4$ & $8.4(5)$ \\
\hline $\mathrm{C} 5-\mathrm{S} 1-\mathrm{C} 11-\mathrm{C} 13$ & $-61.7(3)$ \\
\hline $\mathrm{C} 5-\mathrm{S} 1-\mathrm{C} 11-\mathrm{C} 12$ & $-156.9(3)$ \\
\hline $\mathrm{C} 14-\mathrm{N} 2-\mathrm{C} 12-\mathrm{C} 11$ & $165.7(6)$ \\
\hline $\mathrm{C} 13-\mathrm{N} 2-\mathrm{C} 12-\mathrm{C} 11$ & $15.0(4)$ \\
\hline $\mathrm{C} 13-\mathrm{C} 11-\mathrm{C} 12-\mathrm{N} 2$ & $-14.4(4)$ \\
\hline $\mathrm{S} 1-\mathrm{C} 11-\mathrm{C} 12-\mathrm{N} 2$ & $100.7(4)$ \\
\hline $\mathrm{C} 14-\mathrm{N} 2-\mathrm{C} 13-\mathrm{C} 11$ & $-164.9(6)$ \\
\hline $\mathrm{C} 12-\mathrm{N} 2-\mathrm{C} 13-\mathrm{C} 11$ & $-15.1(4)$ \\
\hline $\mathrm{C} 12-\mathrm{C} 11-\mathrm{C} 13-\mathrm{N} 2$ & $14.3(4)$ \\
\hline $\mathrm{S} 1-\mathrm{C} 11-\mathrm{C} 13-\mathrm{N} 2$ & $-93.9(4)$ \\
\hline $\mathrm{C} 15-\mathrm{N} 3-\mathrm{C} 14-\mathrm{N} 2$ & $172.4(7)$ \\
\hline $\mathrm{C} 15-\mathrm{N} 3-\mathrm{C} 14-\mathrm{S} 2$ & $-0.9(7)$ \\
\hline $\mathrm{C} 12-\mathrm{N} 2-\mathrm{C} 14-\mathrm{N} 3$ & $21.4(9)$ \\
\hline $\mathrm{C} 13-\mathrm{N} 2-\mathrm{C} 14-\mathrm{N} 3$ & $161.4(5)$ \\
\hline $\mathrm{C} 12-\mathrm{N} 2-\mathrm{C} 14-\mathrm{S} 2$ & $-165.3(4)$ \\
\hline $\mathrm{C} 13-\mathrm{N} 2-\mathrm{C} 14-\mathrm{S} 2$ & $-25.3(8)$ \\
\hline $\mathrm{C} 16-\mathrm{S} 2-\mathrm{C} 14-\mathrm{N} 3$ & $-12.7(5)$ \\
\hline $\mathrm{C} 16-\mathrm{S} 2-\mathrm{C} 14-\mathrm{N} 2$ & $173.4(5)$ \\
\hline $\mathrm{C} 14-\mathrm{N} 3-\mathrm{C} 15-\mathrm{C} 16$ & $18.3(10)$ \\
\hline $\mathrm{N} 3-\mathrm{C} 15-\mathrm{C} 16-\mathrm{S} 2$ & $-26.1(10)$ \\
\hline $\mathrm{C} 14-\mathrm{S} 2-\mathrm{C} 16-\mathrm{C} 15$ & $21.0(7)$ \\
\hline $\mathrm{C} 18-\mathrm{O} 5-\mathrm{C} 17-\mathrm{O} 4$ & $-80.2(6)$ \\
\hline
\end{tabular}




$\begin{array}{llll}\mathrm{C} 4-\mathrm{C} 5-\mathrm{C} 6-\mathrm{C} 10 & -171.7(4) & \mathrm{C} 10-\mathrm{O} 4-\mathrm{C} 17-\mathrm{O} 5 & 143.8(4) \\ \mathrm{S} 1-\mathrm{C} 5-\mathrm{C} 6-\mathrm{C} 10 & 3.5(5) & \mathrm{C} 17-\mathrm{O} 5-\mathrm{C} 18-\mathrm{O} 6 & -0.7(9) \\ \mathrm{C} 1-\mathrm{N} 1-\mathrm{C} 6-\mathrm{C} 5 & -108.0(4) & \mathrm{C} 17-\mathrm{O} 5-\mathrm{C} 18-\mathrm{C} 19 & -179.7(5) \\ \mathrm{C} 3-\mathrm{N} 1-\mathrm{C} 6-\mathrm{C} 5 & 4.8(4) & \mathrm{O} 6-\mathrm{C} 18-\mathrm{C} 19-\mathrm{C} 21 & -21.0(12) \\ \mathrm{C} 1-\mathrm{N} 1-\mathrm{C} 6-\mathrm{C} 10 & 73.2(5) & \mathrm{O} 5-\mathrm{C} 18-\mathrm{C} 19-\mathrm{C} 21 & 158.0(9) \\ \mathrm{C} 3-\mathrm{N} 1-\mathrm{C} 6-\mathrm{C} 10 & -174.1(3) & \mathrm{O} 6-\mathrm{C} 18-\mathrm{C} 19-\mathrm{C} 20 & 31.0(10) \\ \mathrm{C} 1-\mathrm{C} 2-\mathrm{C} 7-\mathrm{O} 2 & -50.0(5) & \mathrm{O} 5-\mathrm{C} 18-\mathrm{C} 19-\mathrm{C} 18-\mathrm{C} 19-\mathrm{C} 22 & 98.0(9) \\ \mathrm{C} 3-\mathrm{C} 2-\mathrm{C} 7-\mathrm{O} 2 & 51.5(5) & \mathrm{O} 5-\mathrm{C} 18-\mathrm{C} 19-\mathrm{C} 22 & -83.0(8) \\ \mathrm{C} 1-\mathrm{C} 2-\mathrm{C} 7-\mathrm{C} 8 & -172.6(4) & & \end{array}$

Hydrogen-bond geometry $\left(\AA,{ }^{\circ}\right)$

\begin{tabular}{lllll}
\hline$D-\mathrm{H} \cdots A$ & $D-\mathrm{H}$ & $\mathrm{H} \cdots A$ & $D \cdots A$ & $D-\mathrm{H} \cdots A$ \\
\hline $\mathrm{O} 2-\mathrm{H} 2 A \cdots \mathrm{N} 3^{\mathrm{i}}$ & 0.82 & 2.01 & $2.816(6)$ & 169 \\
$\mathrm{C} 11-\mathrm{H} 11 A \cdots \mathrm{O} 2^{\mathrm{ii}}$ & 0.98 & 2.43 & $3.366(6)$ & 160 \\
\hline
\end{tabular}

Symmetry codes: (i) $x-1, y+1, z$; (ii) $x, y-1, z$. 